\title{
Polarimetric Evidence of the First White Dwarf Pulsar: The Binary System AR Scorpii
}

\author{
David A.H. Buckley ${ }^{1, *(\mathbb{D})}$, Stephen B. Potter ${ }^{1}$, Pieter J. Meintjes ${ }^{2}$, Tom R. Marsh ${ }^{3}$ and \\ Boris T. Gänsicke ${ }^{3}$ \\ 1 South African Astronomical Observatory, Cape Town 7925, South Africa; sbp@saao.ac.za \\ 2 Department of Physics, University of the Free State, Bloemfontein 9301, South Africa; MeintjPJ@ufs.ac.za \\ 3 Department of Physics, University of Warwick, Coventry CV4 7AL, UK; t.r.marsh@warwick.ac.uk (T.R.M.); \\ Boris.Gaensicke@warwick.ac.uk (B.T.G.) \\ * Correspondence: dibnob@saao.ac.za; Tel.: +27-021-460-6286
}

Received: 23 October 2017; Accepted: 15 January 2018; Published: 22 January 2018

\begin{abstract}
The binary star AR Scorpii was recently discovered to exhibit high amplitude coherent variability across the electromagnetic spectrum (ultraviolet to radio) at two closely spaced $\sim 2 \mathrm{~min}$ periods, attributed to the spin period of a white dwarf and the beat period. There is strong evidence (low X-ray luminosity, lack of flickering and absense of broad emission lines) that AR Sco is a detached non-accreting system whose luminosity is dominated by the spin-down power of a white dwarf, due to magnetohydrodynamical (MHD) interactions with its M5 companion. Optical polarimetry has revealed highly pulsed linear polarization on the same periods, reaching a maximum of $40 \%$, consistent with a pulsar-like dipole, with the Stokes $Q$ and $U$ variations reminiscent of the Crab pulsar. These observations, coupled with the spectral energy distribution (SED) which is dominated by non-thermal emission, characteristic of synchrotron emission, support the notion that a strongly magnetic ( 200 MG) white dwarf is behaving like a pulsar, whose magnetic field interacts with the secondary star's photosphere and magnetosphere. Radio synchrotron emission is produced from the pumping action of the white dwarf's magnetic field on coronal loops from the M-star companion, while emission at high frequencies (UV/optical/X-ray) comes from the particle wind, driven by large electric potential, again reminiscent of processes seen in neutron star pulsars.
\end{abstract}

Keywords: pulsars; white dwarfs; polarization

\section{Introduction}

The close binary system AR Scorpii was recently discovered by Marsh et al. [1] to consist of a fast rotating $(\sim 2 \mathrm{~min})$ white dwarf in a $3.6 \mathrm{~h}$ orbit with an M5 red dwarf. Furthermore, the system appears to be spinning down at a rate of $\dot{P} \sim 4 \times 10^{-13} \mathrm{~s} \mathrm{~s}^{-1}$, which is the dominant source of the system's luminosity. This extensive multi-wavelength study, from radio to X-ray wavelengths, showed that the system's SED is dominated by pulsed non-thermal emission, predominantly at the $118 \mathrm{~s}$ beat period of the system. The low X-ray luminosity and lack of evidence for mass transfer (no flickering or broad emission lines) argues strongly against any accretion in the system, although the secondary star is clearly subjected to irradiation from the white dwarf, judging from the strong photometric orbital modulation coupled with the radial velocity motion of the narrow emission lines from the inner face of the secondary. These comprise both optical lines (Balmer and He I) and UV lines (Si IV and He II). The discovery by Buckley et al. [2] that AR Sco is highly linearly polarized (up to $40 \%$ ) and, more importantly, that the polarization is strongly modulated (up to $90 \%$ pulse fraction) on both the spin and beat periods, has strengthened the evidence that AR Sco behaves as a pulsar. The high level of polarization also led Buckley et al. [2] to conclude that the white dwarf is highly magnetic, with a 
field strength as high as $500 \mathrm{MG}$. Various interpretations have been proposed to explain the observed properties, including direct MHD interactions between the magnetic fields of both components [2], possibly producing bow shocks close to the surface of the M-star companion (e.g., [3,4]).

\section{Luminosities}

Marsh et al. [1] determined the distance to AR Sco of $116 \pm 16$ pc from fitting an M5 star template to the observed spectrum and assuming the M-star companion was close to filling its Roche lobe. This led to the derivation of the individual luminosities of the M5 and white dwarf components, which in total is $\sim 4 \times 10^{31} \mathrm{erg} \mathrm{s}^{-1}$. This represents just $\sim 15 \%$ of the total system luminosity, dominated by the modulated non-thermal component, which varies from $0.6-3.6 \times 10^{32} \mathrm{erg} \mathrm{s}^{-1}$. The spin-down power of the system, derived from $\dot{P}$, can be calculated from the formula $L_{\dot{v}}=-4 \pi^{2} I v_{s} \dot{v}_{s}$, where $I$ is the moment of inertia and $v$ the spin frequency. The moment of intertia varies by over 5 orders of magnitude between a neutron star and a white dwarf, leading to spin-down powers of $1.1 \times 10^{28}$ and $1.5 \times 10^{33} \mathrm{erg} \mathrm{s}^{-1}$, respectively. For the observed $\dot{P}$, it is clear that the pulsed luminosity cannot be powered by a spinning down neutron star, since this falls short by about a factor of $\sim 2 \times 10^{4}$ in the required power. On the other hand, a spinning down white dwarf with the observed $\dot{P}$ is capable of explaining the total modulated power in the system.

In mass transferring white dwarf - red dwarf binaries (i.e., cataclysmic variables, or CVs), the dominant source of luminosity comes from accretion. The conclusion (e.g., [1,2]) that the white dwarf has a high magnetic field strength raised the possibility that AR Sco was an asynchronous magnetic $C V$, or intermediate polar, which are typically quite hard ( $k T \sim 10-20 \mathrm{keV})$ and moderately luminous X-ray sources $\left(\sim 10^{31}-10^{33}\right.$ erg s $\left.{ }^{-1}\right)$. In contrast, AR Sco has an X-ray luminosity of $5 \times 10^{30} \mathrm{erg} \mathrm{s}^{-1}$ (from a Swift observation [1]), inconsistent with it being an intermediate polar. In addition, both the optical spectra and the photometric variations are different from that expected for a mass transferring binary (i.e., a CV). Spectral lines in such systems are typically much broader, often multi-component in nature, and are kinematically traced to accreting gas close to the white dwarf, or within its Roche lobe, like in an accretion disk. This is in contrast to the narrow emission lines seen in AR Sco that come from close to the L1 point of the illuminated secondary star [1]. There is also the tell-tale absence of high frequency $(>1 \mathrm{~Hz})$ flickering associated with accretion in cataclysmic variables, in both magnetic and non-magnetic systems. Therefore the conclusion reached by $[1,2]$ is that AR Sco is a detached binary, with no signs of accretion or mass loss through L1, powered predominantly by the spin-down of the magnetic white dwarf.

Finally, the observed low X-ray luminosity is added evidence that AR Sco cannot be a neutron star binary, i.e., a low mass X-ray binary (LMXB). At a distance of only 116 pc, this would make it the nearest and least $X$-ray luminous LMXB, with an observed $L_{X} / L_{o p t}$ ratio of only $\sim 0.04$, quite atypical of LMXBs, whose ratios are typically $\sim 100$.

\section{Polarization Discovery and Behaviour}

High speed photopolarimetry of AR Sco was undertaken on two consecutive nights (14 \& 15 March 2016) using the HIPPO polarimeter on the SAAO 1.9-m telescope. This instrument ([5]) enables all four Stokes parameters to be derived simultaneously, at a sampling rate as high a $10 \mathrm{~Hz}$, using photomultplier tubes. The rapid photon-counting sampling mitigates atmospheric effects and allows data to be binned on any suitable timescale, to suit the desired time resolution or signal to noise. The observations shown here ([2]) were binned such that the intensity $(I)$ is sampled at 1 s intervals, while the other Stokes parameters $(Q, U \& V)$ were binned to $10 \mathrm{~s}$.

In Figure 1 we show the observations from the first night (14 March 2016), which show that the system was strongly linearly polarized, at levels reaching $40 \%$, modulated predominantly on the harmonic of the spin and beat period, as determined from a period analysis [2]. These two nights of observations covered differing orbital phase, namely $\phi \sim 0.07-0.23$ and $0.38-0.85$, respectively, which is the reason for differences in the phase-folded (on the $117 \mathrm{~s}$ spin period) polarization curves, 
as shown in Figure 2 (bottom panels). These clearly show double-peaked polarized intensity variations, modulated with up to $\sim 90 \%$ pulse fraction (on $14 \mathrm{March}$ ). The linear polarization variations, plus the large swing in position angle $\left(\Delta \theta=180^{\circ}\right)$, is a result of the viewing aspect, where the dipole is nearly perpendicular to the rotation axis of the white dwarf.

The difference between the curves on the two nights is a consequence of the different orbital phases of the two observations, which were at an average of $\phi_{\text {orb }} \sim 0.15$ and $\phi_{\text {orb }} \sim 0.64$, respectively. Both the spin and beat modulations combine differently on the two nights, resulting in the variability of the waveforms for the phase-folded observations. Subsequent observations, spanning many orbital cycles, have confirmed the stability of the orbital modulation and hence the amplitudes and phases of the side-band frequencies (work in progress).

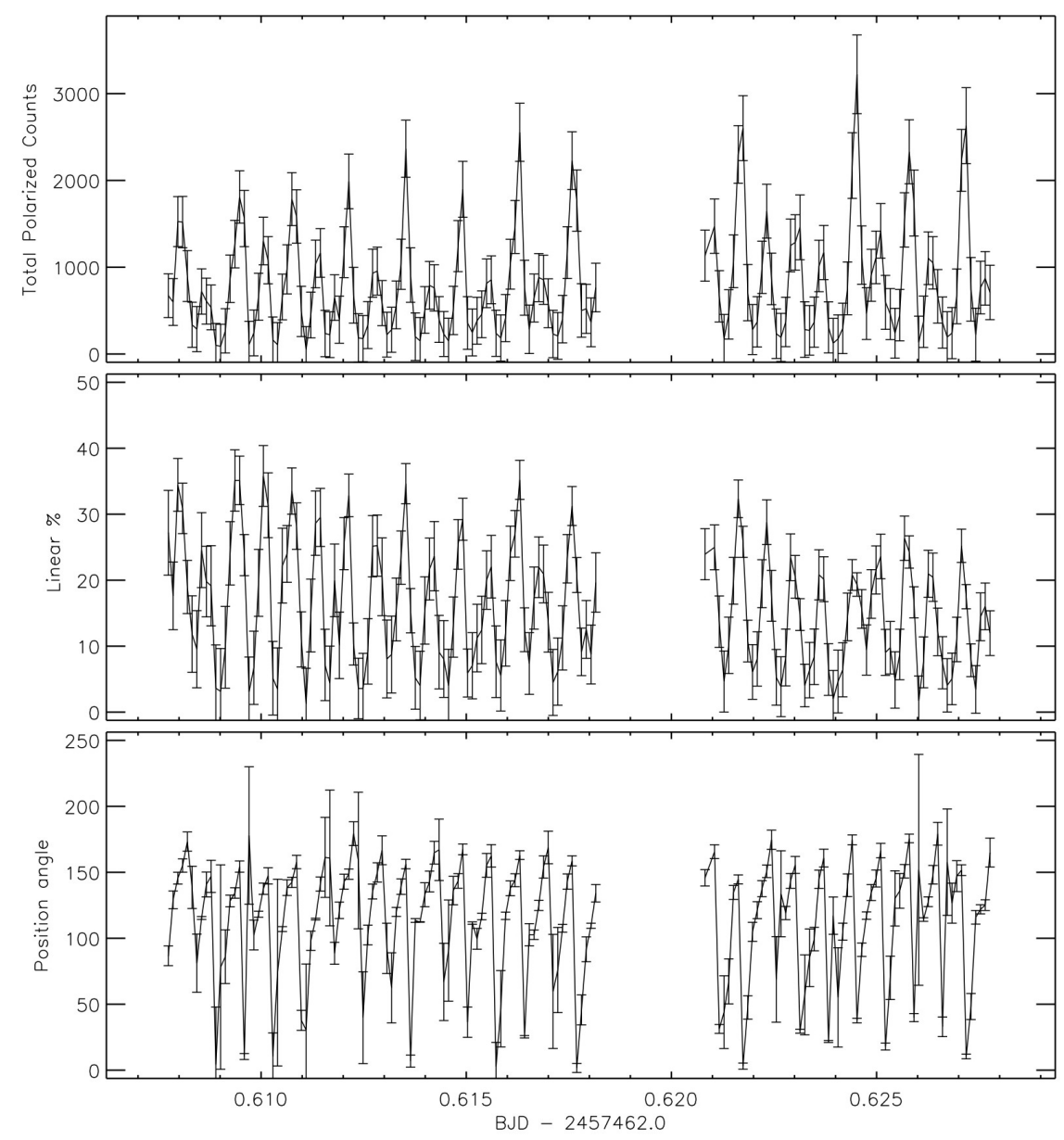

Figure 1. Photopolarimetry of AR Sco covering the band 570-900 nm, taken on 14 March 2016, in $10 \mathrm{~s}$ bins. The panels show, from the top, the total polarized flux (s), degree of linear polarization $(p)$ and position angle of linear polarization $(\theta)$.

In the top panel of Figure 2 we show the spin phase averaged $Q$ and $U$ data, together with the trajectories of their motion in the $Q-U$ plane, which follow counter-clockwise loops, due to the changing magnetic field orientation. On the first night $\left(\phi_{\text {orb }} \sim 0.15\right)$, the main peak maps to the outer loop and the secondary peak maps to the small loop inside it. For the second night $\left(\phi_{\text {orb }} \sim 0.65\right)$, there is an apparent phase change and more complex polarized flux variations (see bottom panel), leading to a different trajectory in the $Q-U$ plane, indicative of changes in the combined white dwarf-red dwarf magnetic field topolgy with respect to our line of sight over the two nights. 
The $Q$ and $U$ variations in AR Sco are qualitatively similar to that seen in the optical polarimetry of the Crab pulsar [6], although for the Crab $p$ and $\theta$ variations show somewhat different morphologies, with more abrupt $\theta$ "swings", possibly a consequence of the lower phase resolution of the AR Sco data. The polarized flux for the Crab shows a duty cycle of $\sim 30 \%$, less that the minimum duty cycle estimated for AR Sco of $\sim 60 \%$. For radio pulsars there are a range of different $\theta$ swing morphologies observed [7], which have been interpreted in terms of a rotating vector model, where the polarization vector $(p, \theta)$ is a projection on the sky of the direction of the magnetic field in the region where the polarized radiation is emitted.
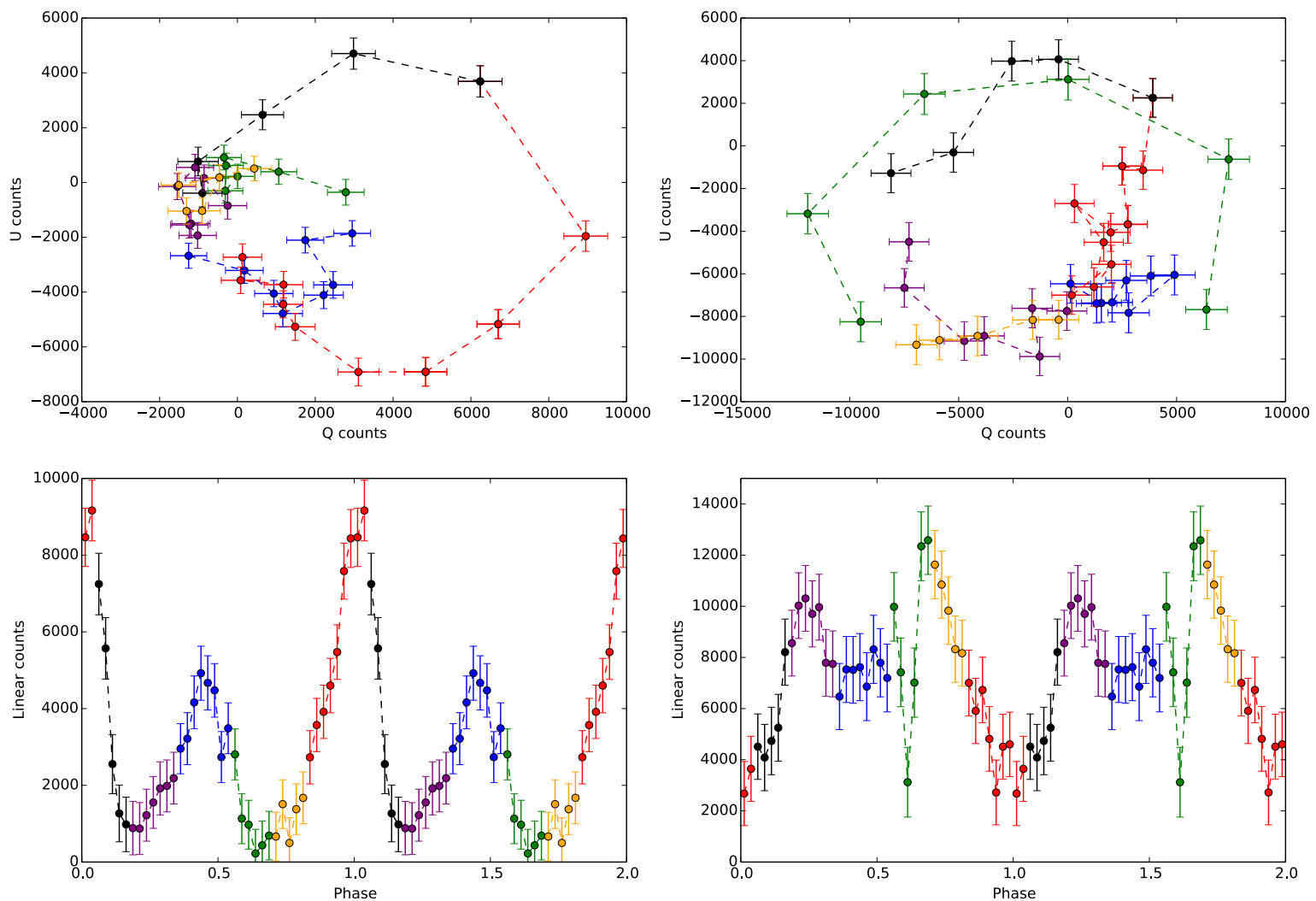

Figure 2. Spin phased variation of the Stokes $Q$ and $U$ parameters (upper panels) and total linearly polarized flux (lower panels) for the red-band (570-900nm) on the two nights 14 (left) and 15 (right) March 2016. The migration of the Stokes $Q$ and $U$ amplitude pairs are shown, plotted every $3 \mathrm{~s}$, with 40 points plotted per spin cycle. Points are colour-coded as in the average phase-folded linearly polarized flux plots. The $Q, U$ pairs follow counter-clockwise trajectories.

\section{Interpretation and Proposed Model}

The strongly pulsed polarized emission in AR Sco is analogous to that observed in pulsars, like the Crab. The ratio of X-ray luminosity to spin-down power for AR Sco, $\alpha=L_{\mathrm{x}} / L_{\mathrm{s}-\mathrm{d}}$, is $\sim 10^{-3}$, implying that most of the luminosity of the system is not produced by accretion of matter, but by spin-down energy loss, just as in a spin-down powered pulsar.

An upper limit on the magnetic dipole strength can be derived assuming that the bulk of the spin-down power is radiated by dipole radiation, which was shown by Buckley et al. [2] to be $~ 500 \mathrm{MG}$. Values this high have been derived for isolated magnetic white dwarfs, which is also within a factor of $2 \times$ of that seen in strongest magnetic cataclysmic variable ( $250 \mathrm{MG})$. An alternative model, assuming that a fraction of the spin-down power is dissipated through a magnetic stand-off shock near the secondary, has led to an estimate of the magnetic field of $\sim 100 \mathrm{MG}$ [4]. If rotational energy is also dissipated through magnetohydrodynamical (MHD) pumping of the secondary star, then a constraint 
can be placed by estimating the MHD power dissipated in the surface layers of the secondary [8]. Dissipation will occur through magnetic reconnection and Ohmic heating, particularly in that part of the secondary star's photosphere which faces the white dwarf. This could contribute to both the observed line emission and the strong orbital photometric modulation, which is at a maximum when the secondary star is at superior conjunction, when it is at the most favourable viewing aspect.

Due to the absence of conducting plasma from mass loss/accretion, it was shown [2] that electric potentials of the order $\Delta V \sim 10^{12} \mathrm{~V}$ can be induced over the distance between the white dwarf and the light cylinder, which has a radius of $6 \times 10^{11} \mathrm{~cm}, \sim 10 \times$ the orbital separation of the two stars. This potential can accelerate electrons to energies of the order of $\gamma_{\mathrm{e}} \sim 10^{6}$, resulting in pulsed and strongly polarized synchrotron emission, possibly up to to X-ray frequencies. The high level of linear polarization and its modulation is consistent with synchrotron emission of relativistic electrons in ordered magnetic fields [2]. The periodic behaviour of the polarization, at both the white dwarf spin and the beat periods, is also consistent with the emission being produced by the interaction of the white dwarf's magnetosphere with the M-dwarf, which explains the beat period as a consequence of orbital modulation.

The spectral energy distribution (SED) in AR Sco [1,4]) shows a $S_{v} \propto v^{\alpha_{1}}\left(\alpha_{1} \sim 1.3\right)$ self-absorbed power law spectral distribution for $v \leq 10^{12}-10^{13} \mathrm{~Hz}$, i.e., at infrared to radio wavelengths. We have suggested [2] that these originate from pumped coronal loops of the nearly Roche lobe-filling secondary star. The magnetic flux tubes of the secondary star are periodically distorted by the fast rotating white dwarf magnetic field, inducing strong field-aligned potentials and synchrotron flares, consistent with the observed power law at $v \leq 10^{12} \mathrm{~Hz}$ and the peak emission at $v_{\text {crit }} \sim 0.3 v_{\text {syn }} \leq 10^{13} \mathrm{~Hz}$. The emission is therefore expected to be pulsed at the beat frequency, consistent with the ATCA observations at 5.5 and $9.0 \mathrm{GHz}$ [1].

At higher frequencies, $v \geq$ few $\times 10^{14} \mathrm{~Hz}$ (optical-UV-X-rays), the SED follows a different $v^{\alpha_{2}}$ power law [1], where $\alpha_{2} \sim-0.2$ (see also [3]). This component is produced by non-thermal synchrotron emission from accelerated charged particles as the magnetic white dwarf dipole interacts with the M-star magnetic field and wind, and can explain the high level of linear polarization observed at optical frequncies.

\section{Discussion}

The high degree of asynchronism in AR Sco $\left(P_{S} / P_{\text {orb }}=0.009\right)$ implies that the white dwarf was spun up, presumably as a result of a previous phase of mass transfer. Another system, AE Aqr, which also harbours a fast spinning $\left(P_{S}=33 \mathrm{~s}\right)$ white dwarf which is spinning down [9], was shown $[10,11]$ to have experienced a previous high mass transfer phase. In this case the secondary star may have shed its outer envelope in a catastrophic run-away mass transfer process, resulting in the white dwarf being spun-up. However, in the case of AR Sco, there is currently no observational evidence to indicate that it has evolved through such an extreme high mass transfer phase. Therefore the evolutionary path which AR Sco has followed is still somewhat of an open question.

The high magnetic field of the white dwarf in AR Sco also presents a significant problem when it comes to spinning up the white dwarf in the first place, since material will tend to be ejected rather than accreted, except at very high accretion rates. This is precisely what the current situation is for AE Aqr, which is acting as a propellor, ejecting rather than accreting material from its companion [9]. Since the observed white dwarf spin-down timescale for AR Sco of $\sim 10^{7}$ years [1,2] is less than the spin-orbit synchronization timescale of $\sim 2.5 \times 10^{8}$ years, calculated for MHD torques alone [2], this implies that most of the spin-down power is dissipated through other channels. The high value of the magnetic field is therefore the reason for the current large spin down rate, through the various mechanisms (e.g., dipole radiation, MHD interactions) which rob the white dwarf of its angular momentum.

AR Sco is a unique object and the best candidate for a white dwarf pulsar-notwithstanding the one crucial point that it is not a neutron star-based on the following attributes: 
- $\quad$ Spin-down powered

- SED dominated by synchrotron emission

- Strongly linearly polarized

- Spin modulated magnetic dipole

- Pulsations are seen from the radio to the ultraviolet (and more recently in X-rays)

- Beamed radiation from relativistic electrons accelerated in a stong electrical potential

- Lorentz factors of $\gamma \sim 400-10^{6}$

We show an artist's impression of AR Sco in In Figure 3.

Further observations, particularly at UV, X-ray and radio wavelengths, will be important in determining the exact nature of the emission mechanisms operating in AR Sco. More extensive time resolved polarimetry, obtained at SAAO during the 2016 and 2017 observing seasons, will also help to constrain the polarized emission models by disentangling the two closely spaced polarized signals, at the spin and beat period, leading to more definitive conclusions regarding geometry. Its proximity in the outskirts of the $\rho$ Ophiuchus molecular cloud also raises the interesting prospects that the system could be a source of EHE $\gamma$-ray emission through charged particle interactions.

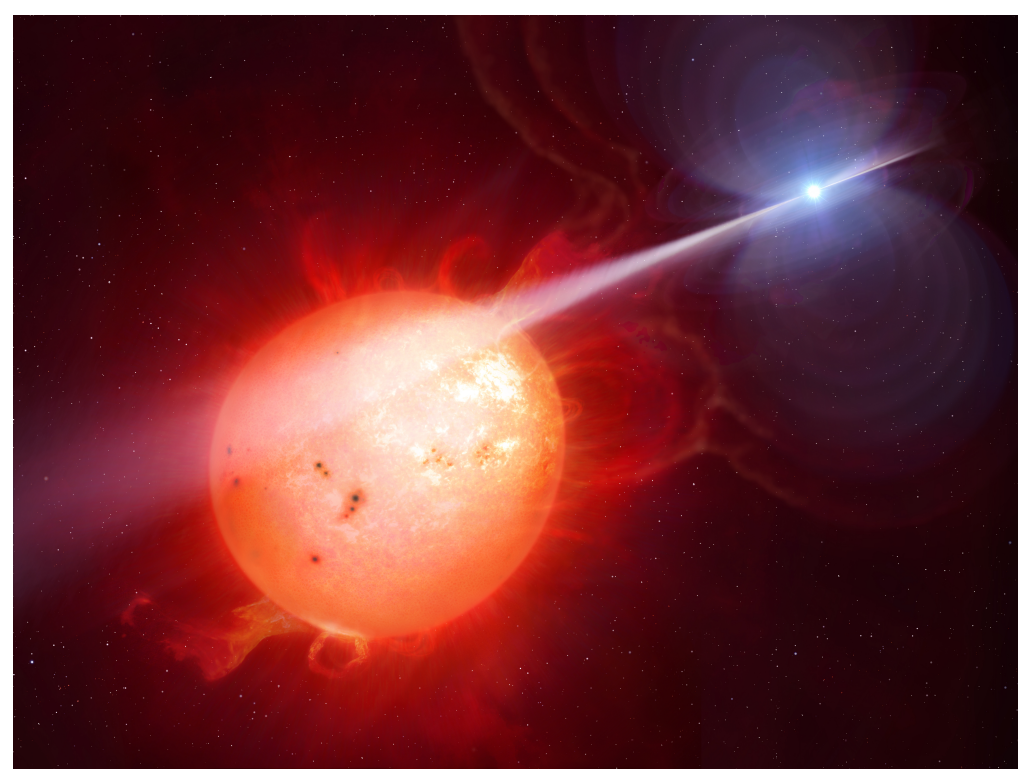

Figure 3. Artist's impession of the white dwarf pulsar system, AR Sco. Credit: Mark Garlick/University of Warwick.

Acknowledgments: D.A.H.B., P.J.M. and S.B.P. were supported by the National Research Foundation of South Africa. T.R.M. was supported by the Science and Technology Facilities Council (STFC) under grant ST/L000733. B.T.G. is supported through European Research Council grant 320964. This work is based on observations obtained at the South African Astronomical Observatory.

Author Contributions: The polarimetric observations were conceived and undertaken by D.A.H.B., who assisted in the analysis and interpretation, participated in the modelling and was primary author of the paper. The modelling work was led by P.J.M. Reductions of the polarimetry was carreid out by S.B.P., who also produced the figures and assisted in interpretation of the results. Initial information on AR Sco, including pre-publication material, was provided by T.R.M. and B.T.G., who also assisted in the interpretation.

Conflicts of Interest: The authors declare no conflict of interest.

\section{References}

1. Marsh, T.R.; Gänsicke, B.T.; Hümmerich, S.; Hambsh, F.J.; Bernhard, K.; Lloyd, C.; Breedt, E.; Stanway, E.R.; Steeqhs, D.T.; Parsons, S.G.; et al. A pulsing, radio emitting white dwarf. Nature 2016, 537, 374-377. 
2. Buckley, D.A.H.; Meintjes, P.J.; Potter, S.B.; Marsh, T.R.; Gänsicke, B.T. Polarimetric evidence of a white dwarf pulsar in the binary system AR Scorpii. Nat. Astron. 2017, 1, 0029.

3. Geng, J.-J.; Zhang, B.; Huang, Y.-F. A model of white dwarf pulsar AR Scorpii. Astrophys. J. Lett. 2016, 831, L10-L14.

4. Katz, J.I. AR Sco: A Precessing White Dwarf Synchronar? Astrophs. J. 2017, 835, 150.

5. Potter, S.B.; Buckley, D.A.H.; O’Donoghue, D.; Remero-Colmenero, E.; O'Connor, J.; Fourie, P.; Evans, G.; Sass, C.; Crause, L.; Still, M.; et al. Polarized QPOs from the INTEGRAL polar IGRJ14536-5522 (=Swift J1453.4-5524). Mon. Not. R. Astron. Soc. 2010, 402, 1161-1170.

6. Słowikowski, A.; Kanbach, G.; Kramer, M.; Stefanescu, A. Optical polarization of the Crab pulsar: Precision measurements and comparison to the radio emission. Mon. Not. R. Astron. Soc. 2009, 397, 103-123.

7. Karastergiou, A.; Johnston, S. Absolute polarization position angle profiles of southern pulsars at 1.4 and 3.1 GHz. Mon. Not. R. Astron. Soc. 2006, 365, 353-366.

8. Meintjes, P.J.; Jurua, E. Secondary star magnetic fields in close binaries. Mon. Not. R. Astron. Soc. 2006, 372, 1279-1288.

9. Ikhsanov, N.R. The pulsar-like white dwarf in AE Aqarii. Astron. Astrophys. 1998, 338, 521-526.

10. Meintjes, P.J. On the evolution of the nova-like variable AE Aquarii. Mon. Not. R. Astron. Soc. 2002, 336, 265-275.

11. Schenker, K.; King, A.R.; Kolb, U.; Wynn, G.A.; Zhang, Z. AE Aquarii: How cataclysmic variables descend from supersoft binaries. Mon. Not. R. Astron. Soc. 2002, 337, 1105-1112.

(C) 2018 by the authors. Licensee MDPI, Basel, Switzerland. This article is an open access article distributed under the terms and conditions of the Creative Commons Attribution (CC BY) license (http://creativecommons.org/licenses/by/4.0/). 